\title{
Situación actual de los estudios de traducción en Filología Española en China: análisis basado en los Trabajos de Fin de Máster y Tesis Doctorales de las universidades chinas (2010-2019) ${ }^{1}$
}

Current Situation of Translation Studies in Spanish Major in China: Analysis based on Postgraduate Dissertations Produced in Chinese Universities (2010-2019)

\section{Yi WANG}

University of International Business and Economics

yi.wang@uibe.edu.cn

\section{Wei Sun}

Universitat Autònoma de Barcelona

felipe@bfsu.edu.cn

Resumen: El presente trabajo revisa los Trabajos de Fin de Máster y Tesis Doctorales relacionados con los estudios de traducción realizados por los estudiantes especializados en Filología Española en China entre 2010 y 2019, los clasifica y los analiza, prestando

1. Financiado por los «Fondos de Investigación Fundamental para las Universidades Centrales» en UIBE (17QD19). (Supported by «the Fundamental Research Funds for the Central Universities» in UIBE [17QD19]). 
mayor atención a sus métodos y objetos de investigación. Trata de descubrir las ventajas y limitaciones de estos trabajos de investigación, las razones que los explican y posibles propuestas de mejora, así como proporcionar algunas sugerencias para el desarrollo de la especialidad de Filología Española en las universidades chinas, especialmente para la formación de posgrado en estudios de traducción.

Palabras clave: universidades chinas; Filología Española; estudios de traducción; Trabajo Fin de Máster; Tesis Doctoral.

\begin{abstract}
This paper examines the postgraduate dissertations related to translation studies in Spanish-language majors in China from 2010 to 2019, classifies and analyses them, focusing on their research methods and research objects. This investigation tries to find out the advantages and limitations of these dissertations, the underlying reasons and possible proposals for future improvement, as well as to provide some suggestions for the development of Spanish studies in Chinese universities, especially in relation to translation studies approaches at postgraduate level.
\end{abstract}

Key words: Chinese universities; Spanish; translation studies; postgraduate dissertations.

\title{
1. INTRODUCCIÓN
}

La traducción, una actividad de comunicación intercultural, posee una historia milenaria. A principios de la segunda mitad del siglo xx, aparecen los primeros estudios sistematizados sobre la traducción y, después del desarrollo en la época fundacional entre los años cincuenta y sesenta, se consolida la Traductología como una disciplina propia (Hurtado Albir 2011, 123-124).

En China, la práctica y teoría de traducción registra un rápido desarrollo en los últimos años del siglo xx, lo que permite que la Traductología se establezca como una disciplina independiente (Xu y Mu 2009, 77). Desde comienzos del nuevo siglo, los estudios de traducción han recibido cada vez mayor atención y la didáctica de la traducción se ha desarrollado rápidamente (Xu y Mu 2009, 88-90).

Con el desarrollo de los estudios de traducción en China, el objeto de investigación ya no se limita a la traducción entre inglés y chino, sino que se ha expandido a más combinaciones lingüísticas. Dado que es uno de los idiomas de trabajo de las Naciones Unidas, el español tiene una importancia insustituible. Así, desde 1979, la Universidad de Estudios Extranjeros de Beijing empezó a impartir formación de posgrado especializada en Filología Española. A partir de 2013, 15 instituciones han obtenido la autorización para otorgar títulos de Máster en dicha especialidad (Zheng y Liu 2014, 602). En cuanto a los estudios de doctorado en Filología Española, en 1996, el Comité de Títulos Académicos del Consejo de Estado aprobó la solicitud de la Universidad de

Yi WANG y Wei SUN Situación actual de los estudios de traducción en Filología Española en China...
CLINA

vol. 6-1, June 2020, 37-50

elSSN: 2444-1961

Ediciones Universidad de Salamanca - CC BY-NC-ND 
Estudios Extranjeros de Beijing para otorgar títulos de doctor. Más tarde, la Universidad de Pekín y la Universidad de Estudios Internacionales de Shanghái también comenzaron a admitir doctorandos en Filología Española. Como una línea de investigación de la Filología Española, los estudios de traducción han atraído la atención de muchos investigadores durante muchos años, y el número de disertaciones ha ido en aumento. En ellas se pone de manifiesto el conocimiento de los jóvenes investigadores chinos en el campo de los estudios de traducción, por lo que constituye un elemento clave para reflejar el desarrollo de la disciplina. Por tanto, para conocer la situación actual de la investigación sobre traducción en el ámbito de la Filología Española en las universidades chinas y la formación de posgrado en Filología Española, nos centraremos en elaborar estadísticas relativas a los Trabajos de Fin de Máster (TFM) y Tesis Doctorales sobre traducción realizados por estudiantes de posgrado de dicha especialidad presentados durante los últimos diez años (2010-2019).

\section{DATOS Y ANÁLISIS}

Hemos recogido datos de las 15 instituciones que ofrecen títulos de Máster en la especialidad de Filología Hispánica, que son las siguientes: la Universidad de Estudios Extranjeros de Beijing (BFSU, por sus siglas en inglés), la Universidad de Beijing (PKU), la Universidad Normal de la Capital (CNU), la Universidad de Estudios Internacionales de Beijing (BISU), la Universidad de Lengua y Cultura de Beijing (BLCU), la Universidad de Economía y Comercio Internacionales (UIBE), la Universidad de Estudios Extranjeros de Tianjin (TFSU), la Universidad de Lenguas Extranjeras de Dalian (DUFL), la Universidad de Jilin (JLU), la Universidad de Estudios Internacionales de Shanghái (SISU), la Universidad de Nanjing (NJU), la Universidad de Estudios Extranjeros de Guangdong (GDUFS), la Universidad de Estudios Internacionales de Sichuan (SISU2)2, la Universidad de Estudios Internacionales de Xi'an (XISU), la Universidad de Estudios Internacionales de Jilin (JISU). Todas ellas tienen competencias para otorgar títulos de Máster y tres de ellas (BFSU, PKU y SISU) títulos de doctor. La muestra total se compone de 175 trabajos de investigación, los cuales han sido recopilados principalmente a través de un barrido de la base de datos de la Unión de Referencia y Consulta de Bibliotecas Nacionales (全国图书馆参考咨询联盟, Quanguo Tushuguan Cankao Zixun Lianmeng) $)^{3}$. Las 15 instituciones arriba mencionadas también nos han proporcionado información complementaria al respecto. A continuación, vamos a analizar dichos trabajos.

2. Como la Universidad de Estudios Internacionales de Shanghái y la Universidad de Estudios Internacionales de Sichuan tienen la misma sigla, para distinguirlas, usamos SISU para referirnos a la primera y SISU2 para la segunda.

3. En este trabajo, en el uso de palabras de origen chino seguimos las normas propuestas por Casas-Tost, Fustegueres i Rosich, Qu, Rovira-Esteva y Vargas-Urpí (2015).

Yi WANG Y Wei SUN Situación actual de los estudios de traducción en Filología Española en China...
CLINA

vol. 6-1, June 2020, 37-50

elSSN: 2444-1961

Ediciones Universidad de Salamanca - CC BY-NC-ND 


\subsection{Situación general de los estudios de traducción en la Filología Española en China}

Encontramos 5 Tesis Doctorales, que representan un 2,86 \% del total de la muestra, y 170 TFM, que representan un 97,14\%. La notable diferencia se debe, en gran medida, a la falta de personal docente. Para la Traductología en general, la falta de profesores es un fenómeno común. Actualmente en China se admiten 110 doctorandos en Traductología cada año, y se forma cada año un número menor que este de doctorandos en dicha disciplina en las universidades de otros países. Pese a que podrán ser profesores en el futuro, el número de profesores con formación especializada en traducción todavía es muy reducido. Por eso, la falta de personal docente no se paliará a corto plazo (Mu 2018, 11).

En el caso de la Filología Española, la mayor parte de los docentes son jóvenes, por lo que faltan profesores con experiencia docente y con título de doctor o títulos profesionales superiores (Zheng y Liu 2014, 598-602). Si analizamos distintas universidades, BFSU tiene seis profesores para dirigir los Trabajos de Fin de Máster, SISU tiene cuatro, y el resto tiene, como mucho, tres, dos o solo uno. Por tanto, a pesar de que el número de instituciones que admiten estudiantes de posgrado va aumentando, el número de estudiantes admitidos en los programas de Máster en Filología Española sigue siendo escaso. En cuanto al título de doctor, solo tres universidades disponen de un programa de posgrado en Filología Española, en los cuales hay en total cinco directores de Tesis Doctorales, pero la mayoría de ellos ya tienen una edad avanzada o ya están jubilados, y faltan profesores que puedan asumir la dirección de Tesis Doctorales en el futuro (Zheng 2015, 67-68). La escasez de personal docente restringe notablemente las posibilidades de admitir y formar a los estudiantes en Filología Española, y, como consecuencia, el número de estudiantes de posgrado, sobre todo de doctorandos, en esta disciplina es reducido. Entre ellos, los que tienen la Traductología como línea de investigación son menos aún. Del conjunto de universidades, solo BFSU ha admitido doctorandos que abordan la traducción en su investigación doctoral. Desde 2012, ninguna universidad tiene oferta de doctorado en Filología Española con una línea de investigación específica centrada en la Traductología.

Además, para la elaboración del presente artículo hicimos un análisis diacrónico del número de trabajos de investigación, que se representa en la Figura 1.

Como se muestra en la figura, a partir de 2014, el número de trabajos de investigación sobre traducción ha ido aumentando constantemente. Eso se debe a que las universidades ofrecen cada vez más plazas en sus programas de Máster. Además, desde 2015, algunas universidades (BFSU, BISU, BLCU, SISU y JISU) empezaron a ofrecer el título de Máster en Traducción e Interpretación (MTI), de perfil profesional, lo que contribuye al aumento de la producción de estudios sobre la traducción.

Tras esto, extrajimos datos desglosados por universidades. Los resultados se presentan en la Figura 2.

Yi WANG Y Wei SUN Situación actual de los estudios de traducción en Filología Española en China..
CLINA

vol. 6-1, June 2020, 37-50

eISSN: 2444-1961

Ediciones Universidad de Salamanca - CC BY-NC-ND 


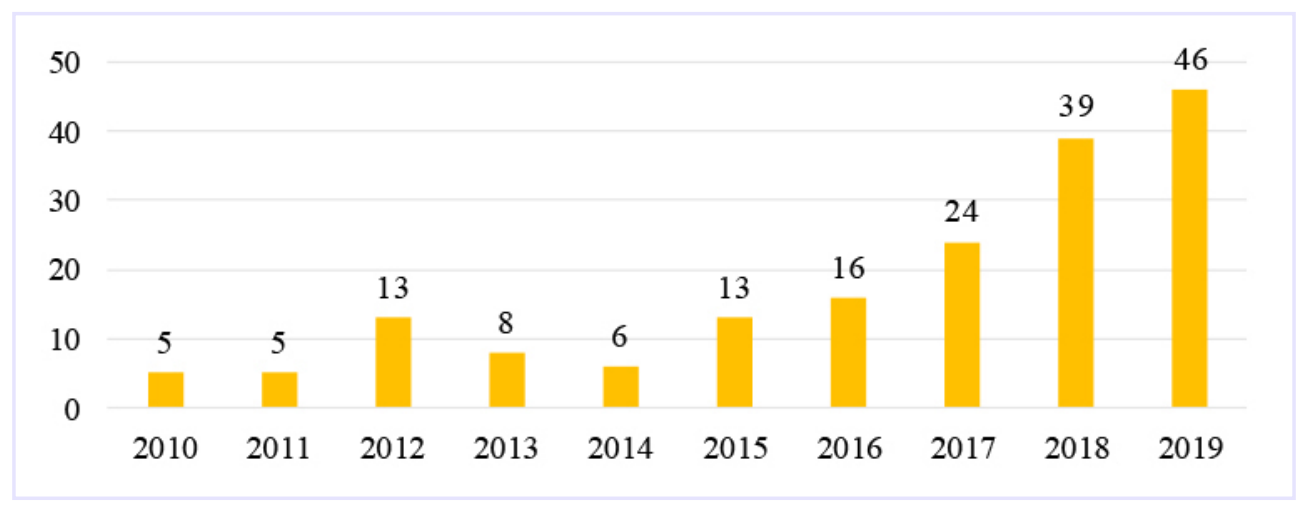

Figura 1. Distribución de los trabajos según año

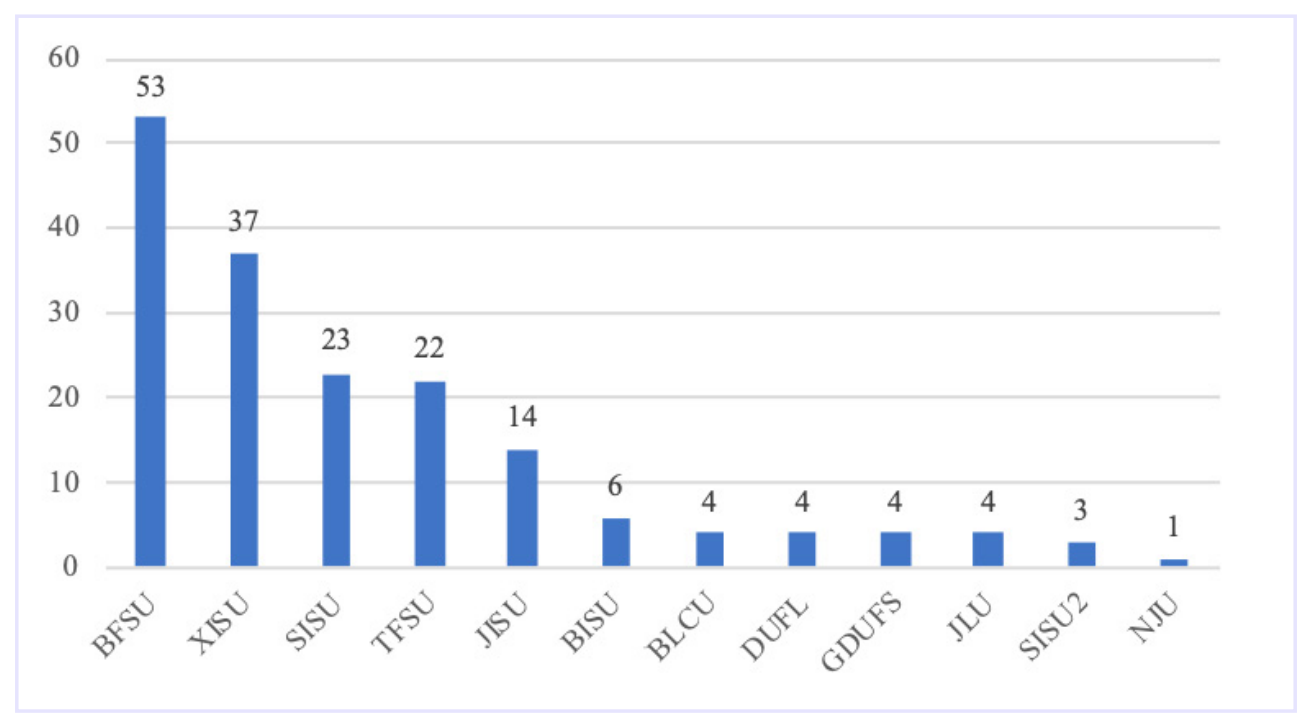

Figura 2. Distribución de los trabajos según institución

Los 175 TFM y tesis proceden de 12 universidades; es decir, no todas las instituciones que ofrecen un Máster en Filología Española tienen la Traductología como línea de investigación. Además, hay mucha diferencia entre la producción académica de las distintas universidades. BFSU ocupa el primer lugar en cuanto a número de TFM y tesis, que representan un 30,29 \% del total, seguida de XISU, SISU y TFSU, que representan el $21,14 \%$, el 13,14 \% y el 12,57 \% respectivamente. Para dar con una explicación a este fenómeno, revisamos la formación del profesorado de estos centros 
y vimos que tienen enfoques diferentes. Por ejemplo, la investigación en PKU, NJY y JLU se centra más en el ámbito de la literatura; en UIBE, por su parte, priman los estudios sobre la economía, política y cultura de los países hispanohablantes; y BFSU, XISU y SISU tienen un mayor número de directores y alumnos en estudios específicos sobre traducción.

\subsection{Clasificación de los TFM y tesis según el objeto de investigación}

Según el objeto de investigación, clasificamos los TFM y tesis en la siguiente tabla:

Tabla 1. TFM y tesis según objeto de investigación

\begin{tabular}{|c|c|c|}
\hline Objeto de investigación & Número & Porcentaje \\
\hline Didáctica de la traducción & 10 & $5,71 \%$ \\
\hline Teoría de la traducción & 5 & $2,86 \%$ \\
\hline Historia de la traducción & 3 & $1,71 \%$ \\
\hline Textos no literarios & 62 & $35,43 \%$ \\
\hline Textos literarios & 49 & $28 \%$ \\
\hline Interpretación & 29 & $16,57 \%$ \\
\hline Informe de traducción/interpretación (MTI) & 17 & $9,71 \%$ \\
\hline
\end{tabular}

A partir de estos datos, podemos observar que los investigadores prefieren estudios basados en textos concretos, sean literarios o no literarios. En cuanto a los temas, los menos tratados son la teoría y la historia de la traducción, mientras que se han hecho muchos trabajos (29) sobre interpretación.

\section{(1) Didáctica de la traducción}

Hay en total 10 estudios, todos los cuales son TFM, sobre la didáctica de la traducción, lo que representa un $5,71 \%$ del total. Estos trabajos abordan principalmente la elaboración de manuales de traducción, la didáctica de traducción en el aula y aspectos generales de la formación de traductores. Los instrumentos de recogida de datos empleados para su elaboración incluyen los cuestionarios, los experimentos y los corpus documentales.

Desde el punto de vista cuantitativo, el número de trabajos sobre didáctica es todavía limitado. Además, cabe señalar que no se han encontrado estudios sobre la competencia traductora, la evaluación de traducciones o la evaluación en el ámbito de la didáctica. 
(2) Teoría de la traducción

El número de trabajos sobre teorías de la traducción es muy pequeño: solo se registran 4 TFM y 1 Tesis Doctoral, lo que supone un porcentaje del 2,86 \% del total. En cuanto a la metodología, limitada por el tema, son trabajos de revisión bibliográfica.

La razón de la escasez de estudios teóricos puede ser, por un lado, tal como señalan Mu y Zou $(2014,15)$, que los estudios teóricos de la traducción en China siempre han seguido la tendencia de la investigación occidental y se ha ralentizado a medida de que se ha producido una desaceleración de los estudios de este campo en Occidente; por otro lado, como los estudios teóricos son más difíciles y exigen un nivel académico más elevado por parte del investigador, sería más adecuado abordar este tipo de estudios en la fase de doctorado. Sin embargo, como mencionábamos anteriormente, dada la falta de personal docente ya comentada, la formación de doctorado en este campo tiene un largo camino por recorrer.

\section{(3) Historia de la traducción}

Los estudios sobre la historia de la traducción son aún más escasos: con solo 3 TFM dedicados a este tema, constituye el tema menos tratado en la muestra analizada. Son estudios descriptivos basados en un corpus documental y se ocupan principalmente de estudiar la traducción y difusión de obras literarias españolas en China, así como el desarrollo de las actividades vinculadas a la traducción en China a lo largo de la historia.

Esta escasez se puede explicar por la falta de materiales de estudio; ya que muchos materiales históricos ya están estudiados, sería cada vez más difícil encontrar nuevas perspectivas (Mu y Zou 2014, 16). Además, tras la revisión de los programas docentes de las distintas universidades, no encontramos asignaturas o cursos relacionados con la historia de traducción, lo que también puede ser una causa de la escasez de estudios al respecto.

\section{(4) Traducción de textos no literarios}

Del conjunto de trabajos recopilados, los más numerosos son los que tratan de la traducción de textos no literarios. Con 60 TFM y 2 tesis, representan un 35,43 \% del total y se distribuyen de manera uniforme durante los años, lo que pone de manifiesto que constituye un tema de destacado interés para los investigadores.

En muchos casos, son estudios basados en tipos de texto concretos, pero procedentes de diferentes campos temáticos y entornos socioprofesionales, por ejemplo, noticias, anuncios, discursos políticos y diplomáticos, textos audiovisuales, textos especializados (jurídicos, económicos), entre otros. También hay muchos trabajos sobre cuestiones que plantean dificultades específicas en la traducción, como la traducción de los nombres propios, el lenguaje de internet, los pronombres de tratamiento, los falsos amigos, los términos de la medicina tradicional china, o sobre aspectos más generales como la sintaxis o la interculturalidad, para lo cual se recogen ejemplos de fuentes diversas.

Yi WANG Y Wei SUN Situación actual de los estudios de traducción en Filología Española en China..
CLINA

vol. 6-1, June 2020, 37-50

elSSN: 2444-1961

Ediciones Universidad de Salamanca - CC BY-NC-ND 
La mayoría de los estudios son descriptivos y comparativos y los resultados obtenidos son principalmente cualitativos, pero también hay estudios basados en datos cuantitativos a partir de corpus.

(5) Traducción de textos literarios

La traducción literaria es otro tema que interesa mucho a los investigadores, ya que encontramos 49 TFM que se ocupan de la traducción de textos literarios, los cuales representan un $28 \%$ del total y se distribuyen de manera uniforme durante los años.

En la mayoría de los casos, son estudios basados en alguna obra literaria concreta y que investigan sobre las técnicas o estrategias empleadas, o que realizan un análisis o evaluación de las traducciones. También hay estudios sobre aspectos más específicos, como la variación lingüística, el humor, las rimas, entre otros. Los géneros literarios más estudiados son la novela (88 \%) y la poesía (12\%). Las obras estudiadas se recogen en la siguiente tabla:

Tabla 2. TFM y tesis sobre traducción de textos literarios por obras

\begin{tabular}{|c|c|}
\hline Obras & Número \\
\hline La fortaleza asediada (围城, Weicheng) & 2 \\
\hline Sueño en el pabellón rojo (红楼梦, Honglou Meng) & 5 \\
\hline El ciruelo en el vaso de oro (金瓶梅, Jin Ping Mei) & 1 \\
\hline Cuentos fantásticos del estudio del charlatán (聊斋志异, Liaozhai Zhiyi) & 1 \\
\hline Viaje al oeste (西游记, Xiyou Ji) & 1 \\
\hline A la orilla del agua (水浒传, Shuihu Zhuan) & 1 \\
\hline Romance de los Tres Reinos (三国演义, Sanguo Yanyi) & 1 \\
\hline Contar nuevo de historias viejas (故事新编, Gushi Xinbian) & 1 \\
\hline La casa de té (茶馆, Chaguan) & 1 \\
\hline Vivir (活着, Huozhe) & 3 \\
\hline Rana (蛙, Wa) & 2 \\
\hline El suplicio del aroma de sándalo (檀香刑, Tanxiang Xing) & 1 \\
\hline Don Quijote de la Mancha (堂吉诃德, Tang Ji He De) & 4 \\
\hline Cien años de soledad (百年孤独, Bainian Gudu) & 4 \\
\hline El amor en los tiempos del cólera (霍乱时期的爱情, Huoluan Shiqi de Aiqing) & 1 \\
\hline Poesía de la China antigua & 6 \\
\hline
\end{tabular}

Yi WANG Y Wei SUN Situación actual de los estudios de traducción en Filología Española en China... 
En la tabla podemos observar que:

a) Aunque hay variedad en cuanto a las obras estudiadas, también se puede observar una preferencia por algunas, entre ellas Sueño en el pabellón rojo, Don Quijote de la Mancha o Cien años de soledad.

b) El corpus seleccionado para el estudio lo constituyen principalmente obras clásicas antiguas, en contraste con la menor atención que reciben las modernas y contemporáneas.

c) El número de obras chinas es mucho mayor que el de las obras españolas o hispanoamericanas; es decir, los investigadores prefieren estudiar la traducción inversa en vez de la directa.

(6) Interpretación

Se registran 29 estudios sobre la interpretación (17 TFM y 2 Tesis Doctorales), lo que representa un porcentaje del 16,57 \% del total. Los estudios proceden principalmente de las universidades que ofrecen títulos de MTI, sobre todo BFSU, SISU y JISU. La concentración de trabajos académicos demuestra que estas instituciones han avanzado significativamente tanto en profundidad como en cuanto a variedad en la investigación en interpretación. La distribución temporal de los estudios se presenta en la Figura 3:

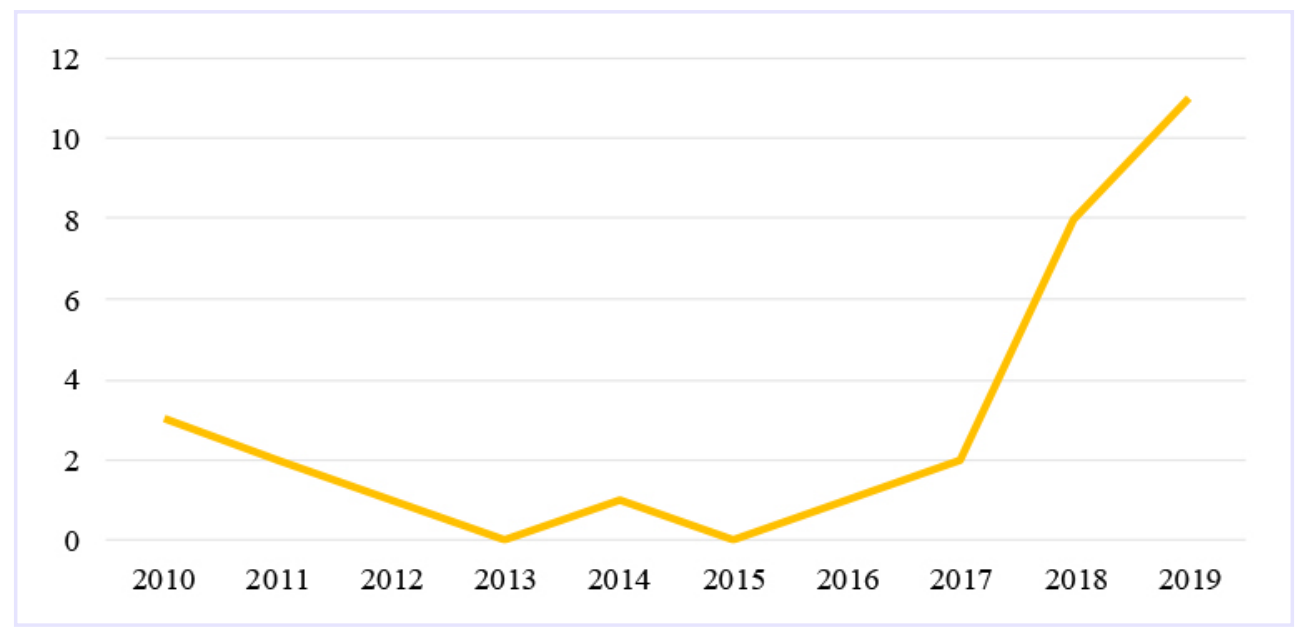

Figura 3. Distribución de los trabajos de interpretación por año

En la Figura 3 podemos observar una tendencia a la baja antes del año 2015, lo que puede explicarse porque había poco personal con capacidad para dirigir trabajos de investigación, que, por otra parte, estaba a punto de jubilarse. Sin embargo, con la implantación de títulos de Máster en Traducción e Interpretación (MTI) a partir de 
2015, se nota una fuerte tendencia al alza desde 2017, lo que se explica con el hecho de que la duración del MTI es de dos años y los primeros estudiantes comenzaron a graduarse en ese año.

En cuanto al tema, muchos estudios tratan sobre distintos aspectos relevantes en la interpretación, por ejemplo, la transmisión de determinados tipos de información, la segmentación de los discursos en unidades de traducción, el uso de técnicas como la reformulación o la simplificación, entre otras. También hay estudios que aplican algunas teorías al ámbito de la interpretación, como la teoría de la relevancia o las teorías funcionalistas, entre otras. Estos trabajos se basan principalmente en grabaciones o vídeos de interpretaciones reales y presentan resultados cualitativos. En la mayoría de los casos, analizan interpretaciones consecutivas en el ámbito político o comercial, tanto del chino al español como del español al chino. También hay estudios sobre otras modalidades, por ejemplo, la interpretación de enlace y la interpretación telefónica.

En lo que se refiere a la metodología, hay estudios descriptivos de carácter cualitativo, pero también se registran estudios empíricos basados en cuestionarios, experimentos y entrevistas.

\section{(7) Informe de traducción / interpretación}

Al terminar la carrera, los estudiantes de MTI tienen dos opciones: pueden escribir un trabajo académico, igual que los de Máster Académico, o hacer un informe a partir de una experiencia propia de traducción o interpretación. En total 17 estudiantes optaron por esta última opción, entre los cuales 11 se centran en la traducción escrita y 6 en la interpretación.

Entre estos 11 estudiantes que se decantan por la traducción, 10 eligen textos literarios y el alumno restante traduce un texto gubernamental. En cuanto a la direccionalidad, 8 estudiantes hacen traducción directa y 3 traducción inversa. El modelo empleado es parecido en todos los casos: realizan la labor de traducción siguiendo la orientación de una teoría y luego hacen comentarios y reflexiones sobre las traducciones hechas.

Además, 6 alumnos de los que realizaron un trabajo de este tipo tenían experiencia como intérpretes de conferencia. De ellos, 5 se centran en la interpretación consecutiva y el sexto, en la interpretación simultánea. Los informes de interpretación tienen un enfoque distinto: en la mayoría de los casos, tratan alguna cuestión concreta con relevancia en el proceso de la interpretación, como las notas, la repetición, las pausas. Además, analizan los apuntes tomados en sus propias interpretaciones o las grabaciones de las mismas para identificar los problemas encontrados, así como para plantear posibles soluciones.

Yi WANG Y Wei SUN Situación actual de los estudios de traducción en Filología Española en China.. 


\section{PROPUESTAS DE MEJORA}

Tras la clasificación, análisis estadístico y cualitativo de los 175 trabajos de investigación, vemos que, en los últimos años, sobre todo durante los tres últimos, van surgiendo cada vez un mayor número de estudios que versan sobre la traducción en la especialidad de Filología Española, lo que pone de manifiesto un creciente interés de los investigadores por la traducción y una mayor variedad en los objetos de estudio y la metodología adoptada. Es un fenómeno alentador. Sin embargo, los datos recogidos también reflejan algunas limitaciones:

\section{(1) Objeto de estudio}

Los estudios de traducción en Filología Española tratan una gama variada de temas, como la didáctica de la traducción, la teoría de la traducción, la traducción de textos literarios y no literarios, la interpretación, entre otros. En la gran mayoría de los casos, son estudios basados en textos, pertenecientes a diferentes campos temáticos y entornos socioprofesionales. Los textos se estudian como productos de la traducción. Sin embargo, en la Traductología actual, los estudios no se reducen a los productos; también se investiga la cognición en la traducción, la adquisición de la competencia traductora, los aspectos profesionales de la traducción y la interpretación, la influencia y posibilidades de las tecnologías de información y comunicación (TIC) aplicadas a la traducción, la traducción automática, entre otros. Los citados son temas muy poco tratados en los trabajos que encontramos, que, a pesar de su gran interés académico, muestran aún vacíos que cubrir en la investigación.

Además, el análisis pone de manifiesto que se pueden abrir nuevas perspectivas de investigación. Por ejemplo, en el caso de estudios sobre la traducción literaria, se nota una preferencia hacia las obras clásicas de la China antigua, sobre todo Sueño en el pabellón rojo, que es objeto de estudio en cinco trabajos. Sin embargo, otras obras más recientes reciben menor atención en los estudios. En los trabajos sobre la interculturalidad, destaca en términos cuantitativos la reflexión sobre los problemas de traducción aparejados a las connotaciones de los colores en las distintas culturas, las frases hechas y los refranes, que son temas ya muy tratados en la Traductología. No obstante, se registran muy pocos estudios sobre las religiones o las artes, que también son componentes importantes de la cultura.

(2) Marco teórico

En su estudio específico sobre el marco teórico de Tesis Doctorales en Traductología, Mu y Li (2016) han encontrado cuatro problemas principales:

a) Falta de marco teórico: en algunos trabajos no hay ningún marco teórico, por lo que en el análisis falta el apoyo de la teoría;

b) Marco teórico inútil: en algunos estudios, existe una sección dedicada al «marco teórico»; sin embargo, la teoría es demasiado general como para aplicarse

Yi WANG Y Wei SUN Situación actual de los estudios de traducción en Filología Española en China...
CLINA

vol. 6-1, June 2020, 37-50

elSSN: 2444-1961

Ediciones Universidad de Salamanca - CC BY-NC-ND 
al analizar cuestiones concretas, o solo hay una presentación panorámica de varias teorías, sin aplicarlas después en la fase de análisis de manera sistemática;

c) Marco teórico innecesario: en estos casos, las teorías empleadas son innecesarias, en el sentido de que podría llegarse a la misma conclusión sin ellas, o simplemente se buscan ejemplos para corroborar las teorías existentes;

d) Confusión entre marco teórico y modelo de investigación (Mu y Li 2016, 9495).

Comprobamos que estos problemas también están presentes en los estudios de traducción en Filología Española que hemos analizado. Además, en la bibliografía consultada en los trabajos analizados, vemos que hay más obras en chino que en otros idiomas, y, además, hay más monografías que artículos de revistas académicas. Por tanto, puede deducirse que algunas de las teorías empleadas son antiguas y que las tendencias actuales de la disciplina no quedan reflejadas de manera representativa en estos estudios.

\section{(3) Metodología}

En la nueva era, con el desarrollo de la ciencia y la tecnología, la metodología de investigación en el ámbito de la traducción ha ido enriqueciéndose. Las TIC ya se aplican en numerosos estudios traductológicos en Occidente. En la Traductología se nota un claro desarrollo de los estudios empíricos, basados en datos cuantitativos. Sin embargo, del análisis se colige que, entre los trabajos recopilados, son escasos los estudios empíricos basados en datos cuantitativos, y más escasos aún los que emplean las TIC.

Para abrir nuevas vías de investigación, hace falta el esfuerzo tanto de los estudiantes como de los profesores. Por una parte, los profesores deben actualizar constantemente sus conocimientos y reforzar la orientación a los estudiantes a lo largo de todo el proceso de investigación, por ejemplo, ayudándolos a elegir temas viables, novedosos y de interés investigador, ofreciendo asignaturas sobre metodología de la investigación, recursos informáticos y herramientas de documentación, así como sobre las teorías y tendencias actuales de la disciplina. Por otra parte, los estudiantes deben ampliar sus lecturas, tanto en chino como en otros idiomas, y prestar especial atención a las revistas académicas de alto impacto, para ponerse al día de las tendencias actuales y novedades en la disciplina. Además, sería necesario reforzar la práctica de la redacción académica, con el fin de que el estudiante se familiarice con el lenguaje académico y mejore su expresión escrita, tanto en chino como en español.

Yi WANG Y Wei SUN Situación actual de los estudios de traducción en Filología Española en China... 


\section{CONCLUSIÓN}

A través del análisis realizado de los Trabajos de Fin de Máster y Tesis Doctorales de las universidades chinas sobre estudios de traducción en Filología Española, se puede concluir que el número de estos trabajos académicos va creciendo en la última década, lo que pone de manifiesto el desarrollo de esta especialidad y el interés cada vez mayor de los estudiantes por este terreno. Sin embargo, también existen algunos problemas, por ejemplo, en el marco teórico de muchos trabajos. Además, a pesar de la gran variedad de los objetos de estudio, se observa una notable preferencia al estudio sobre la traducción literaria, basándose en el análisis de alguna obra concreta. En cuanto a la metodología, se nota la escasez de los trabajos empíricos. Hace falta abrir nuevas perspectivas en los estudios de investigación.

Actualmente se está experimentando en China un auge en el desarrollo de la especialidad de Filología Española, y hay cada vez más universidades que ofrecen estudios en esta especialidad. Cabe esperar que en el futuro más estudiantes puedan seguir profundizando sus estudios en la fase de Máster y doctorado, y que muchos de ellos opten por realizar estudios sobre la traducción. Por lo tanto, en la medida en que nos enfrentamos a nuevos desafíos en la formación de estudiantes, hacen falta más estudios específicos que traten de buscar nuevas vías que puedan impulsar el desarrollo de los estudios de traducción en Filología Española en el futuro.

\section{BIBLIOGRAFÍA}

Casas Tost, Helena; Fustegueres I Rosich, Sílvia; Qu, Xianghong; Rovira-Esteva, Sara y Vargas-UrPí, Mireia. 2015. Guía de estilo para el uso de palabras de origen chino. Madrid: Adeli Ediciones.

HURTADO AlBiR, Amparo. 2011. Traducción y Traductología. Introducción a la Traductología. Madrid: Ediciones Cátedra.

Mu, Lei (穆雷). 2018. «Fanyixue Xuekejianshe de Tansuo (翻译学学科建设的探索) [Exploración sobre el desarrollo de la Traductología como disciplina]». Zhongguo Fanyi (中国翻译). Chinese Translators Journal 6: 9-11.

Mu, Lei (穆雷) y Xixi LI (李希希). 2016. «Fanyixue Boshilunwen zhongde «Lilun Kuangjia» Wenti Yanjiu (翻译学博士学位论文中的《理论框架》问题研究) [Sobre el «marco teórico» en las Tesis Doctorales en Traductología]». Zhongguo Waiyu (中国外语). Foreign Languages in China 13 (6): 94-101.

Mu, Lei (穆雷) y Bing Zou (邹兵). 2014. «Zhongguo Fanyixue Yanjiu Xianzhuang de Wenxian Jiliang Fenxi (1992-2013): Dui Liangnasidi Jin 700 Pian Boshi Lunwen de Kaocha (中国翻 译学研究现状的文献计量分析 (1992-2013) - - 对两岸四地近700篇博士论文的考察) [Un análisis bibliométrico de la producción de Tesis Doctorales sobre traducción en dos décadas en China: sobre el desarrollo de la Traductología en el país]). Zhongguo Fanyi (中国翻 译). Chinese Translators Journal 4: 14-20.

Yi WANG Y Wei SUN Situación actual de los estudios de traducción en Filología Española en China..
CLINA

vol. 6-1, June 2020, 37-50

elSSN: 2444-1961

Ediciones Universidad de Salamanca - CC BY-NC-ND 
Xu, Jun (许钧) y Lei Mu (穆雷). 2009. Zhongguo Fanyi Yanjiu (1949-2009) (中国翻译研究 (1949-2009) ) [Estudios sobre traducción en China (1949-2009)]. Shanghai: Shanghai Waiyu Jiaoyu Chubanshe.

ZHENG, Shujiu (郑书九) y Yuanqi LIU (刘元祺). 2014. «Woguo Gaodeng Yuanxiao Xibanyayu Zhuanye Shizi Zhuangkuang Diaoyan (我国高等院校西班牙语专业师资状况调研) [Situación actual de los profesores de español en las instituciones de educación superior de China]». Waiyu Jiaoxue yu Yanjiu (外语教学与研究). Foreign Language Teaching and Research 46 (4): 596-604.

ZHENG, Shujiu (郑书九). 2015. Quanguo Gaodeng Yuanxiao Xibanyayu Jiaoyu Yanjiu (全国高等院 校西班牙语教育研究) [Estudio sobre la enseñanza del español en las instituciones de educación superior en China]. Beijing: Waiyu Jiaoxue yu Yanjiu Chubanshe. 